\title{
Minimizing Surgical Skin Incision Scars with a Latex Surgical Glove
}

\author{
Adam D. Schaffner
}

Received: 25 December 2012/Accepted: 31 December 2012/Published online: 23 February 2013

(C) Springer Science+Business Media New York and International Society of Aesthetic Plastic Surgery 2013

Level of Evidence $V$ This journal requires that authors assign a level of evidence to each article. For a full description of these Evidence-Based Medicine ratings, please refer to the Table of Contents or the online Instructions to Authors www.springer.com/00266.

The authors describe a technique to minimize incision length and tissue trauma. Both of these goals are shared by all patients and surgeons. The authors should be commended for developing a technique that is practical and cost effective. Surgeons reading this article should carefully consider the potential for a petroleum-based ointment to cause an adverse reaction (e.g., granuloma) and may consider using an aqueous-based lubricant instead. Adhesive dressings containing iodine may cause an allergic reaction or irritant dermatitis. Therefore, surgeons should consider using a dressing that does not contain iodine. Because of the potential for patients to have an adverse reaction to latex or powder, surgeons should consider using a latex-free, powder-free glove. Surgeons suturing the material should inspect the needle after it penetrates the material to ensure that no material is on the needle tip to avoid embedding a foreign body into the skin or soft tissue.

Optimally, a device will be developed that has many of the advantages of a glove but is available in multiple prefabricated sizes to accommodate different incision lengths, would not require suturing or would require sutures through preformed holes to avoid the potential of embedding material into the skin or soft tissue, and would be radiopaque so it may be identified via imaging should a piece of the material inadvertently break off.

Conflict of interest The author has no conflicts of interest to disclose.

\footnotetext{
A. D. Schaffner $(\bowtie)$

60 East 56th Street, Second Floor, New York, NY 10022-3350, USA

e-mail: drschaffner@drschaffner.com
} 